\title{
Gestión de riesgos de costes de posconstrucción en edificios residenciales en alquiler
}

\section{Risk management of post-construction costs in residential rental buildings}

\author{
$\underline{\text { P. Fernández-Valderrama }}{ }^{(*)}$, J.J. Moyano ${ }^{(*)}$, M.R. Chaza ${ }^{(*)}$
}

\section{RESUMEN}

El coste de posconstrucción de los edificios representa una parte fundamental del coste en su ciclo de vida, por tanto su control es fundamental para lograr la sostenibilidad económica de un inmueble.

Esta investigación propone un modelo para identificar, analizar y evaluar el riesgo de incrementos de coste de posconstrucción en edificios residenciales destinados al alquiler. Para su desarrollo recurrimos a valoraciones de expertos y a la experimentación del modelo en diferentes inmuebles. Empleamos las técnicas de entrevista semiestructurada (83 en total) y método Delphi, con la particularidad en este último de sustituir los formularios de consulta por entrevistas, incrementando así la calidad de los datos.

El modelo propone una escala para graduar el nivel de riesgo del edificio, comprendido entre mínimo-máximo de 36 a 109. Los resultados de la investigación ponen de manifiesto que a partir del valor 75 el nivel de riesgo se considera inasumible.

Palabras clave: Gestión de riesgos, evaluación de riesgos, costes de posconstrucción, edificios residenciales en alquiler, entrevistas semiestructuradas, método Delphi.

\section{ABSTRACT}

The post-construction cost of the buildings represents an essential part of its life cycle cost and therefore its control is fundamental to achieve the economic sustainability of a building.

The present research proposes a model to identify, analyse and assess the risk of increases of post-construction cost in residential buildings for rental. To develop it, we turn to experts' assessments and the experimentation of the model in different buildings. We use the semi-structured interview (a total of 83) as well as the Delphi method: in this last one, inquiry forms are replaced with interviews, so data quality are increased.

The model proposes a scale to adjust the building risk level, which is between a minimum of 36 and a maximum of 109. The research results reveal that from the value of 75, the risk level is considered unacceptable.

Keywords: Risk management, risk assessment, post-construction cost, residential rental buildings, semi-structured interviews, Delphi method.

(*) Universidad de Sevilla (España)

Persona de contacto/Corresponding author: pfvalde@us.es (P. Fernández-Valderrama).

ORCID: http://orcid.org/oooo-0oo2-8964-5766 (P. Fernández-Valderrama); http://orcid.org/oooo-0oo2-2186-6159

(J.J. Moyano); http://orcid.org/oooo-0oo2-1791-80o8 (M.R. Chaza).

Cómo citar este artículo/Citation: Fernández-Valderrama, P.; Moyano, J.J.; Chaza, M.R. (2019). Gestión de riesgos de costes de posconstrucción en edificios residenciales en alquiler. Informes de la Construcción, 71(555): e302. https://doi.org/10.3989/ic.63759.

Copyright: (C) 2019 CSIC. Este es un artículo de acceso abierto distribuido bajo los términos de la licencia de uso y distribución Creative Commons Reconocimiento 4.0 Internacional (CC BY 4.0). 


\section{INTRODUCCIÓN}

En los últimos tiempos se hace cada vez más explícito la extensión del concepto de sostenibilidad de las edificaciones, además de a la perspectiva ambiental, a la social y económica. Las tendencias en la gestión de los edificios se encaminan a disponer de herramientas que permitan evaluar diferentes opciones de proyecto basadas en una eficiencia económica medioambientalmente sostenible (1), por lo que cada vez es más necesario considerar en fase de preconstrucción los costes asociados a la fase de uso de los edificios, así como llevar a cabo una gestión eficiente de los mismos.

El control eficaz de activos es esencial para la mejora de los resultados de las organizaciones, alcanzar valor y lograr un equilibrio entre costo y riesgo. Para que este control sea eficaz es fundamental una gestión de los activos basada en riesgo contribuyendo así a la eficiencia operacional y el logro de los objetivos (2). Por su parte la norma ISO 15686-5: Edificaciones y bienes inmuebles construidos - Planificación de la vida útil - Parte 5: Coste del ciclo de vida (3), pone de manifiesto que los costes correspondientes a la fase que comienza tras la construcción del edificio, o fase de posconstrucción (operación, mantenimiento y final de vida), son los que representan un mayor peso dentro del ciclo de vida completo. Esto pone de manifiesto la trascendencia de identificar el riesgo de incrementos de costes en la fase de posconstrucción de las edificaciones para tomar decisiones informadas relativas a sostenibilidad, viabilidad y eficiencia. La presente investigación se centra en la gestión de riesgos asociados a los costes de posconstrucción de edificaciones residenciales plurifamiliares destinadas al alquiler.

Resulta frecuente que concepto de riesgo tiene diferentes significados para diferentes personas, variando con el punto de vista, las actitudes y la experiencia, siendo visto a veces como un concepto abstracto cuya medición es difícil (4). Según el Project Manager Institute (5) riesgo es todo evento o condición incierta que, si sucede, tiene un efecto sobre, al menos, un objetivo del proyecto. En esta investigación el riesgo es un suceso potencial: los incrementos que puedan experimentarse en los costes de posconstrucción del edificio que, si suceden, pueden afectar el objetivo de mantener estos costes tan bajos como sea razonablemente posible (6).

En general los riesgos han de ser gestionados mediante algún tipo de proceso estructurado. Aunque existen diferentes modelos para su gestión, estos coinciden en las etapas que comprende el proceso: identificación, análisis, valoración y tratamiento. La finalidad principal de la investigación es proponer una herramienta para identificar y analizar el origen de los incrementos de costes de posconstrucción, valorar su repercusión, aportando para ello una escala de graduación, y proporcionar información para su tratamiento.

Son habituales las referencias a que la gestión de riesgos es efectiva cuando se lleva a cabo en las fases iniciales del proyecto $(7,8,9,10)$. El empleo de la herramienta propuesta se prevé en la fase de preconstrucción del edificio, lo que permitirá poner en marcha acciones para materializarlo con menor riesgo. Quiere decirse con ello que las características y circunstancias que rodean el proceso de materialización del edificio en esa fase inicial, pueden determinar resultados muy positivos para el control de los costes de posconstrucción del patrimonio inmobiliario en renta.
La propuesta se suscribe al ámbito geográfico de la Comunidad Autónoma de Andalucía en España. No obstante, es adaptable a otros contextos geográficos y tipos de edificios. También pretende ser una aportación a la gestión de riesgos en el contexto del fomento de alquiler de viviendas, dado el cambio de paradigma que parece estar produciéndose en el régimen de tenencia de los inmuebles en los últimos años en España. Este cambio se refleja en los últimos Planes Estatales de Vivienda en España $(11,12)$, que tienen como uno de sus objetivos el fomento del alquiler de viviendas, a diferencia de los anteriores, cuyos esfuerzos se dirigían a la construcción de vivienda protegida.

El destinatario de la propuesta es el promotor del edificio, su explotador o cualquier agente interesado en conocer el nivel de riesgo: usuarios, inversores, financiadores, etc.

\section{ANTECEDENTES}

La literatura sobre modelado de riesgos en construcción es muy extensa. Desde la primera aparición en 1960 del concepto de riesgo en la bibliografía específica del sector hasta la actualidad, se ha producido una intensificación y sofisticación en su modelado y evaluación, aunque a pesar de ello hay una brecha significativa entre la teoría y su uso práctico (13).

La gestión de riesgos en construcción se asocia más habitualmente a la fase de construcción y especialmente en proyectos de obra civil de gran envergadura y complejidad. Según Akintoye y MacLeod (14) el análisis y la gestión de riesgos en la construcción no se utilizan ampliamente debido a la falta de conocimientos al respecto. Para De Camprieu et al. (15) muchos de los modelos empleados no han sido ampliamente adoptados en la práctica debido a su complejidad. Hwang et al. (16) o Marcelino-Sádaba et al. (17) también refieren los problemas de su aplicación en pequeños proyectos.

Para solventar estas cuestiones Tauron (13) propone el empleo de algún tipo de herramienta analítica sencilla que utilice el coste del riesgo como medida común y la experiencia profesional. En los últimos años han surgido nuevas tendencias que proponen la actitud colaborativa activa entre todas las partes (18), la flexibilidad para la adaptación a las condiciones cambiantes de los riesgos (19) o la atención plena a todo tipo señales de riesgo, basada en una preocupación por el fracaso, las sorpresas y los errores que en cualquier momento pueden presentarse (20).

Por todo ello creemos que es necesario fomentar el empleo formal de técnicas de gestión del riesgo en obras de edificación de cualquier tamaño y complejidad. La presente aportación se desarrolla en esta línea y propone una metodología cuya puesta en práctica y adaptación a las condiciones cambiantes de los riesgos, no reviste complejidad y se basa en la experiencia profesional de expertos del sector de la construcción.

\section{METODOLOGÍA Y DESARROLLO DE LA INVESTIGACIÓN}

La metodología seguida ha consistido en las siguientes fases:

- Analizar el estado de la cuestión relativo a riesgo en el sector de la construcción y a costes posconstrucción.

- Identificar y ponderar variables que definen el riesgo en los edificios objeto de la investigación. Los principales elemen- 
tos de entrada para la investigación se han basado en datos históricos, experiencia, retroalimentación, previsiones y valoraciones de un grupo de expertos. Para identificar el riesgo se ha empleado el método Delphi con la singularidad de sustituir los cuestionarios de consulta a los participantes por entrevistas semiestructuradas. En esta particularidad se invirtió una gran cantidad de tiempo para completar todas las entrevistas, por lo que hubo que ajustar el número de expertos que intervinieron en cada actividad. Las entrevistas realizadas a lo largo de la investigación permitieron aumentar la calidad de los datos obtenidos, ya que se pudo observar el grado de implicación de los expertos y se obtuvo una gran cantidad de información complementaria para el desarrollo del trabajo.

- Modelar el riesgo mediante una formulación que permitiese obtener un valor de riesgo del edificio.

- Experimentar el funcionamiento de la formulación en cuatro casos de estudio. Los experimentos se han llevado a cabo con cada experto en formato de entrevista semiestructurada.

- Interpretar los resultados de valoración del riesgo. Los valores obtenidos se han asociado a una escala de niveles de riesgo expresados en lenguaje natural del tipo, bajo, medio, medio-alto, etc. Para ello se ha contado con el grupo de expertos, con los que se han mantenido nuevas entrevistas.

Los anteriores pasos se llevaron a cabo en diferentes actividades de trabajo. Su contenido y las técnicas empleadas para la obtención de datos se indican en la tabla 1.

\subsection{Identificación de las variables de riesgo}

La propuesta considera 27 variables de riesgo. Para su obtención se partió de una relación inicial de 21 variables, obtenida en base a la revisión bibliográfica, la experiencia y bases de datos propias de los autores de la investigación. El proceso seguido para obtener esta relación inicial comenzó identificando un amplio número de cuestiones relacionadas con los incrementos de coste de posconstrucción o circunstancias generadoras del mismo, tales como usos del inmueble, tolerancia al riesgo y actitudes de los usuarios, tipos de materiales empleados, tipo de mantenimiento llevado a cabo, geometría del edificio y cantidad de supervisión llevada a cabo durante la construcción, entre otras. Como resultado de esta acción se obtuvo un conjunto de 87 elementos. Con vistas a que estos pudieran ser tratados de forma eficiente de forma posterior por el grupo de expertos, se llevó a cabo un exhaustivo proceso de análisis que dio como resultado la agrupación de estos elementos en las 21 variables. Esta relación fue sometida a la valoración de los expertos en sucesivas sesiones de trabajo empleando la técnica Delphi para llegar a un consenso, con la particularidad de sustituir los formularios de consulta por entrevistas semiestructuradas al objeto de aumentar la calidad de los datos. El resultado de esta fase fue la relación consensuada de las 27 variables.

A lo largo de las diferentes actividades y fases de trabajo de la investigación se contó con 21 expertos. El formato de entrevista semiestructurada supuso invertir gran cantidad de tiempo. En ocasiones la duración de las sesiones fue de varias horas, teniendo que recurrir a diferentes jornadas de trabajo para concluirlas. Esto unido a la deslocalización geográfica de los expertos y a que las sesiones se llevaron a cabo en 8 actividades de trabajo repartidas a lo largo de la investigación, motivó que el grueso de las entrevistas se prolongara durante 16 meses. Se trató de fidelizar a los expertos para poder contar con ellos durante todo este período, aunque fue necesario llevar a cabo sustituciones respecto del panel inicial de 21 componentes. Estas sustituciones no fueron aleatorias, sino que se produjeron principalmente en las primeras sesiones, al observar las dificultades por parte de determinados expertos para participar de forma continuada en las diferentes actividades de trabajo a desarrollar a lo largo la investigación.

Para la elección de los colaboradores se adoptó el criterio de que dispusieran de amplia experiencia y conocimiento transversal del sector de la edificación así como ser expertos en diferentes áreas de influencia en la investigación. La media de años de experiencia de los participantes es de 33 y sus características se muestran en la tabla 2.

El empleo de entrevistas semiestructuradas tiene como objetivo incrementar la calidad de los datos. Permite comprobar el grado de implicación de los participantes y la comprensión del contexto de la investigación. Kuo y Lu (21) y Rostami et al. (22), en sus estudios acerca de los riesgos asociados a los proyectos de construcción, emplean este tipo de entrevistas basadas en un reducido grupo de expertos.

Tabla 1. Contenido de las actividades de trabajo con expertos

\begin{tabular}{|c|c|}
\hline Actividades de trabajo & Técnica-Objetivo \\
\hline $\begin{array}{l}\text { 1. Identificación del riesgo. Grupo } 1^{\circ} \text { de entrevistas semiestructura- } \\
\text { das con } 12 \text { expertos. }\end{array}$ & \multirow{3}{*}{$\begin{array}{l}\text { Técnica: Método Delphi y entrevistas semiestructuradas con } \\
\text { expertos (en total } 34 \text { entrevistas). } \\
\text { Objetivo: Obtener una relación de variables de riesgo ponderadas, } \\
\text { en base a la cual se establece una formulación que cuantifique el } \\
\text { riesgo de un edificio. }\end{array}$} \\
\hline $\begin{array}{l}\text { 2. Identificación del riesgo. Grupo } 2^{\circ} \text { de entrevistas semiestructura- } \\
\text { das con } 12 \text { expertos. }\end{array}$ & \\
\hline $\begin{array}{l}\text { 3. Identificación del riesgo. Grupo } 3^{\circ} \text { de entrevistas semiestructura- } \\
\text { das con } 10 \text { expertos. }\end{array}$ & \\
\hline $\begin{array}{l}\text { 4. Valoración del riesgo en edificio } 1 \text { con } 11 \text { expertos. } \\
\text { 5. Valoración del riesgo en edificio } 2 \text { con } 10 \text { expertos. } \\
\text { 6. Valoración del riesgo en edificio } 3 \text { con } 10 \text { expertos } \\
\text { 7. Valoración del riesgo en edificio } 4 \text { con } 5 \text { expertos. }\end{array}$ & $\begin{array}{l}\text { Técnica: Entrevistas semiestructuradas con expertos para aplica- } \\
\text { ción de la formulación (en total } 36 \text { entrevistas). } \\
\text { Objetivo: Obtener valores de riesgo que permitan clasificarlo. Veri- } \\
\text { ficar la consistencia de la relación de variables y del proceso seguido } \\
\text { hasta el momento. }\end{array}$ \\
\hline $\begin{array}{l}\text { 8. Clasificación de los niveles de riesgo y determinar las implicacio- } \\
\text { nes de cada nivel de riesgo con } 13 \text { expertos. }\end{array}$ & $\begin{array}{l}\text { Técnica: Entrevistas semiestructuradas con expertos (en total } 13 \\
\text { entrevistas). } \\
\text { Objetivo: Asociar los valores numéricos de riesgo obtenidos a una } \\
\text { escala de niveles de riesgo expresada en lenguaje natural. Especi- } \\
\text { ficar las repercusiones de cada nivel de riesgo y las acciones para } \\
\text { reducirlo. }\end{array}$ \\
\hline
\end{tabular}


Tabla 2. Características de los expertos participantes

\begin{tabular}{|l|r|}
\hline No de expertos participantes en la investigación & 21 \\
\hline $\begin{array}{l}\text { No mínimo previsto de expertos participantes en } \\
\text { cada actividad }\end{array}$ & 10 \\
\hline Ámbitos de actuación profesional (no de expertos): & \\
\hline Diseño en edificación-construcción & 7 \\
\hline Dirección de obras & 11 \\
\hline Promoción inmobiliaria & 5 \\
\hline Rehabilitación/Restauración de edificaciones & 2 \\
\hline Gestión de inmuebles & 2 \\
\hline Mantenimiento de edificaciones & 3 \\
\hline Administración de fincas & 1 \\
\hline Gestión Integral de la Edificación & 1 \\
\hline Peritación de siniestros en edificación & 2 \\
\hline Media de años de experiencia laboral & 33 \\
\hline $\begin{array}{l}\text { Son docentes universitarios-investigadores además } \\
\text { de profesionales }\end{array}$ & 8 \\
\hline Titulaciones universitarias (no de expertos): & \\
\hline $\begin{array}{l}\text { Arquitectura Técnica/ Aparejador/Ingeniería en Edifica- } \\
\text { ción }\end{array}$ & 6 \\
\hline Arquitectura & 4 \\
\hline Ingeniería Industrial & 1 \\
\hline Ingeniería Técnica de Minas & 1 \\
\hline Derecho & 1 \\
\hline Historia & 2 \\
\hline Doctor/a & 1 \\
\hline Catedrático/a & \\
\hline
\end{tabular}

En esta fase de identificación del riesgo se parte de las 21 variables iniciales y se llevan a cabo tres grupos de entrevistas semiestructuradas para obtener una relación ampliada y consensuada de variables ponderadas. Estas se refieren a conceptos susceptibles de producir incrementos significativos en la cuantía del coste de posconstrucción del edificio. La escala de ponderación utilizada es la de Likert (23), con la particularidad de añadir la posibilidad de optar por el valor o, que se refiere a que la variable no tiene relación con los incrementos de coste. $\mathrm{Al}$ objeto de ajustar el orden de las magnitudes a la hora de cuantificar el riesgo total, donde los valores de riesgo de cada variable se multiplican por su ponderación, estas ponderaciones en la escala de rango o- 5 se homogeneizan dividiendo cada uno de sus valores, entre el mínimo valor de ponderación obtenido por una variable:

Ponderación $_{\text {i FINAL }}=\frac{\text { Ponderación }_{\text {i INICIAL (ESCALA o-5) }}}{\text { Valor de la mínima ponderación }}$

Para este proceso los expertos han tenido en consideración que:

- El coste de posconstrucción se considera desde la puesta en funcionamiento del edificio hasta el final de su vida útil.

- El coste de posconstrucción incluye todo el generado por diferentes conceptos: mantenimiento, limpieza, reparaciones, reposiciones, reformas, mejoras, impuestos y tasas, seguros, consumos energéticos y de recursos, personal, costes de gestión, etc.

- Estos costes son independientes del agente al que corresponda el pago de los mismos.
Tabla 3. Variables ponderadas de riesgo

\begin{tabular}{|c|c|}
\hline Grupos de variables & Ponderación \\
\hline \multicolumn{2}{|l|}{$\begin{array}{l}\text { Variables relativas al diseño y programa } \\
\text { del edificio }\end{array}$} \\
\hline 1. Eficiencia de las instalaciones del edificio. & 1,45 \\
\hline 2. Aislamiento de la envolvente del edificio. & 1,43 \\
\hline 3. Geometría del edificio. & 1,12 \\
\hline $\begin{array}{l}\text { 4. Accesibilidad en las labores de limpieza y } \\
\text { mantenimiento (mantenibilidad). }\end{array}$ & 1,27 \\
\hline $\begin{array}{l}\text { 5. Acabados, materiales y sistemas cons- } \\
\text { tructivos a emplear en las fachadas del } \\
\text { edificio. }\end{array}$ & 1,55 \\
\hline $\begin{array}{l}\text { 6. Acabados, materiales y sistemas cons- } \\
\text { tructivos a emplear en el edificio, excepto } \\
\text { fachadas. }\end{array}$ & 1,42 \\
\hline 7. Elementos singulares en el inmueble. & 1,09 \\
\hline 8. Complejidad de la ejecución de obra. & 1,28 \\
\hline 9. Zonas comunes especiales. & 1,33 \\
\hline 10. Dotaciones del edificio. & 1,34 \\
\hline \multicolumn{2}{|l|}{$\begin{array}{l}\text { Variables relativas a la calidad de la cons- } \\
\text { trucción }\end{array}$} \\
\hline $\begin{array}{l}\text { 11. Idoneidad de las empresas que intervie- } \\
\text { nen en la ejecución. }\end{array}$ & 1,52 \\
\hline 12. Idoneidad del proyectista. & 1,54 \\
\hline $\begin{array}{l}\text { 13. Idoneidad de la dirección facultativa de } \\
\text { las obras y otros agentes intervinientes. }\end{array}$ & 1,53 \\
\hline $\begin{array}{l}\text { 14. Revisión y supervisión llevada a cabo } \\
\text { durante la elaboración del proyecto. }\end{array}$ & 1,31 \\
\hline $\begin{array}{l}\text { 15. Supervisión global llevada a cabo sobre } \\
\text { aspectos técnicos del proyecto. }\end{array}$ & 1,26 \\
\hline $\begin{array}{l}\text { 16. Supervisión adicional llevada a cabo } \\
\text { durante la construcción. }\end{array}$ & 1,36 \\
\hline \multicolumn{2}{|l|}{$\begin{array}{l}\text { Variables relativas al promotor y explota- } \\
\text { dor del edificio y al programa }\end{array}$} \\
\hline $\begin{array}{l}\text { 17. Compromiso extra-económico por parte } \\
\text { del promotor. }\end{array}$ & 1,45 \\
\hline $\begin{array}{l}\text { 18. Compromiso económico por parte del } \\
\text { promotor. }\end{array}$ & 1,47 \\
\hline $\begin{array}{l}\text { 19. Previsiones técnicas, organizativas y } \\
\text { económicas sobre el mantenimiento del } \\
\text { edificio y su gestión. }\end{array}$ & 1,54 \\
\hline \multicolumn{2}{|l|}{ Variables relativas al uso y usuarios } \\
\hline 2o. Intensidad de uso. & 1,44 \\
\hline 21. Grado de rotación de usuarios. & 1,23 \\
\hline $\begin{array}{l}\text { 22. Nivel social, cultural, económico y cívico } \\
\text { de los usuarios. }\end{array}$ & 1,32 \\
\hline \multicolumn{2}{|l|}{$\begin{array}{l}\text { Variables relativas a cuestiones inheren- } \\
\text { tes al edificio y su entorno }\end{array}$} \\
\hline $\begin{array}{l}\text { 23. Grado de protección y singularidad arqui- } \\
\text { tectónica del inmueble. }\end{array}$ & 1,11 \\
\hline 24. Ubicación. & 1,00 \\
\hline 25. Condiciones climáticas. & 1,40 \\
\hline 26. Características geotécnicas del suelo. & 1,24 \\
\hline $\begin{array}{l}\text { 27. Coyuntura y estructura política, económi- } \\
\text { ca y social general. }\end{array}$ & 1,25 \\
\hline
\end{tabular}

A lo largo del proceso de investigación, y a través del método Delphi llevado a cabo durante las actividades de trabajo 1, 2 y 3 (tabla 1), el número de variables se amplió a 27 (tabla 3) respecto de las 21 de partida. 


\subsection{Modelado del riesgo}

Lu y Yan (24) dividen los sistemas de evaluación del riesgo percibido en dos tipos. El primer tipo, los directos, engloba los métodos tradicionales donde el riesgo se cuantifica en base a preguntas a expertos referentes al nivel de riesgo de determinadas variables. El segundo tipo engloba los basados en el Teorema de la Utilidad Esperada, donde se calcula el producto de la probabilidad y el impacto del riesgo consultados a los expertos y asociando las respuestas a escalas numéricas.

En el modelo de la investigación, el nivel de riesgo se cuantifica en base a dos parámetros: la importancia relativa de las variables (Im) respecto a las demás, o ponderación, y la repercusión (Rp) de los posibles incrementos experimentados en la cuantía de los costes de posconstrucción.

De esta manera el nivel de riesgo parcial aportado por cada variable $\left(\mathrm{R}_{\mathrm{i}}\right)$ se modela según la ecuación [1], como producto de su importancia relativa (Im) o ponderación, por su repercusión (Rp):

$$
\mathrm{R}_{\mathrm{i}}=\operatorname{Im} \times \mathrm{Rp}
$$

El riesgo global del edificio (R) es según la ecuación [2], la suma de los riesgos parciales:

$$
\mathrm{R}=\sum \mathrm{R}_{\mathrm{i}}
$$

La importancia del riesgo ( $\mathrm{Im})$, o ponderación de la variable (tabla 3), se considera un parámetro fijo en la formulación de la ecuación [1]. La repercusión (Rp) de cada variable es una valoración de los expertos en cada edificio analizado, considerando:

- El conjunto de características que rodea el proceso de materialización del edificio. Si no existe disponible información sobre las características del edificio, debido al momento en el que se lleva a cabo la valoración, esta se refiere al nivel de incertidumbre relativo a la repercusión. A mayor incertidumbre, mayor riesgo. Otra opción es establecer hipótesis-objetivo sobre las características que el edificio ha de cumplir, quedando condicionado el nivel de riesgo calculado a su cumplimiento. Una finalidad adicional de estos análisis preliminares de riesgo es aportar recomendaciones sobre requisitos o especificaciones de diseño.

- La asiduidad con que puede presentarse el incremento de coste y la severidad del incremento. A mayor asiduidad y severidad, mayor repercusión.

- El comportamiento holístico de las variables, ya que unas pueden compensar las repercusiones de otras. Esta valoración del riesgo en presencia de múltiples factores de riesgo presenta complejidad, aunque es una tarea habitual. Los estudios de Dawson et al. (25) sugieren que un medio de abordar esta complejidad es confiar en las percepciones de riesgo global. Los autores indican que tal enfoque puede proporcionar un atajo cognitivo para identificar cuándo la acción de precaución puede ser necesaria en respuesta a una combinación de múltiples factores de riesgo y vulnerabilidad.

En los modelos de gestión de riesgos a menudo se establecen dos tipos de valoración del riesgo: cualitativa y cuantitativa. Cuando se disponen de datos estadísticos suficientes se emplean las escalas cuantitativas. No obstante, mucha de la información relacionada con el análisis de riesgos no es numérica, más bien se expresa como palabras o frases en un lenguaje natural (26). En el sector de la construcción, donde a menudo no se dispone de datos estadísticos suficientes, se considera adecuado utilizar las escalas cualitativas. La norma ISO 31010:2009 Gestión del riesgo-Técnicas de apreciación del riesgo (27) indica que la apreciación cualitativa del riesgo se define indicando niveles tales como alto, medio y bajo. En la investigación se opta por una escala de este tipo, traduciéndose los tres niveles a números: riesgo bajo equivale a 1, medio a 2 y alto a 3, no obstante los investigadores emplean habitualmente diferentes opciones. Cervone (28) emplea una escala de tres niveles, estableciendo los equivalentes numéricos fijados por Kendrick (29) de 1, 3 y 5 puntos. Zou et al. (7) indican que se pueden asignar diferentes valores para los parámetros del cálculo, alto puede tomar un valor de 1, medio el o,5 y bajo el o,1.

\subsection{Valoración del riesgo en 4 edificios}

Según Nassim (30) para comprender un fenómeno en situación de incertidumbre es necesario entender los extremos, frente a la opción de descartar lo extraordinario y centrarse en lo "normal”. Siguiendo esta línea, los experimentos de valoración llevados a cabo se encaminaron a verificar si en dos edificios con características de riesgo eminentemente bajo y alto respectivamente, los valores obtenidos se correspondían con los niveles mínimo y máximo posibles según la metodología diseñada. Estos niveles se obtienen al asignar a cada variable un riesgo bajo $\mathrm{y}$ alto respectivamente, que se traducen en unas puntuaciones de 1 y 3 en cada caso. Por lo que al ser la ponderación (Im) un factor fijo, aplicando las ecuaciones propuestas: $\mathrm{Ri}=\mathrm{Im} \times \mathrm{Rp}$ [1] y $\mathrm{R}=\sum \mathrm{R}_{\mathrm{i}}$ [2] se obtienen, para las puntuaciones de Rp de 1 y 3 , unos valores totales de riesgo de 36 y 109 respectivamente.

Para verificar estos valores de riesgo situados en los extremos, el equipo de investigación selecciona dos edificios reales ya construidos y bien conocidos profesionalmente por el equipo. El criterio de selección es que los inmuebles presenten características de riesgo bajo y alto respectivamente. A efectos de la experimentación se modifican y añaden ciertas características respecto de las originales, buscando que el nivel de riesgo sea, de forma global y a priori, eminentemente bajo y alto respectivamente. Estas características son a las que se referían las variables: geometría; presencia de elementos singulares; experiencia de los agentes intervinientes en el proceso: promotor, diseñador, constructor, etc.; dotación de instalaciones; accesibilidad para los trabajos de mantenimiento; etc. Durante el desarrollo del experimento de valoración, se observa a través de la entrevistas que el nivel de riesgo percibido por los expertos coincide con el incorporado a los dos inmuebles, es decir, eminentemente bajo y alto respectivamente.

Una vez realizadas las entrevistas y recabadas las valoraciones de todos los participantes, se calcula la media aritmética, obteniendo el edificio 1 una puntuación de riesgo de 41,89 y el edificio 2 de 73,47. Los valores mínimo y máximo posibles según las ecuaciones [1] y [2] son 36 y 109 respectivamente. Por lo que el valor de 41,89, está en el entorno de riesgo mínimo: 36. Sin embargo 73,47 dista del riesgo máximo 109, situándose en el entorno de 72,50; valor medio entre el riesgo mínimo y máximo. Esta circunstancia llama la atención, ya que se observa durante las entrevistas, que el riesgo de este edificio es percibido por los entrevistados como eminentemente alto y sin embargo la valoración del riesgo se sitúa en el punto medio del rango 36-109. 
En base a estos resultados se procede a experimentar con un nuevo edificio. En esta ocasión también se parte de un inmueble real y se ajustan sus características para incrementar el nivel de riesgo respecto al edificio anterior.

Siguiendo el mismo proceso de entrevistas, obtenidos los resultados y calculada la media aritmética de las valoraciones, la puntuación de riesgo del edificio 3 es 73,61; muy similar a la del edificio 2: 73,47 y muy distante del riesgo máximo 109.

De nuevo, a la vista de los resultados se experimenta con un nuevo edificio, en este caso una variante del caso anterior, ajustando nuevamente las características para incrementar el nivel de riesgo. El número de participantes en esta ocasión fue menor, ya que los resultados obtenidos verifican índices muy similares a los precedentes: los tres últimos edificios son diseñados para los experimentos de valoración de riesgo con características de riesgo eminentemente alto, y se observa que este mismo nivel alto es así percibido por los expertos durante los experimentos, y sin embargo los valores obtenidos se sitúan en el entorno de valor medio entre 36 y 109. La puntuación de riesgo en este último edificio es de 75,92. El resumen de los resultados en los cuatro experimentos se indica en la tabla 4.

Tabla 4. Resultados valoraciones de riesgo

\begin{tabular}{|l|c|}
\hline Casos de estudio & Valor del riesgo \\
\hline Edificio 1 & 41,89 \\
\hline Edificio 2 & 73,47 \\
\hline Edificio 3 & 73,61 \\
\hline Edificio 4 & 75,92 \\
\hline Riesgo mínimo (ecuaciones [1] y [2]) & 36,00 \\
\hline Riesgo máximo (ecuaciones [1] y [2]) & 109,00 \\
\hline
\end{tabular}

\subsection{Análisis e interpretación de los resultados}

Tras los resultados obtenidos se llevan a cabo las siguientes actuaciones:

- Verificar si los niveles de riesgo extremos, alto y bajo, incorporado en el diseño de las características de los edificios, se corresponden al mismo nivel de riesgo percibido de forma global por los expertos en los experimentos de cálculo del valor de riesgo.

- Verificar la correlación existente entre los niveles de riesgo global percibido por cada experto en cada edificio y los valores de riesgo obtenidos por los respectivos expertos.

\subsubsection{Correspondencia nivel de riesgo incorporado al} edificio - nivel de riesgo percibido por los expertos

En esta etapa, llevada a cabo en la actividad de trabajo 8 (tabla 1), se solicita a los expertos una valoración del riesgo global percibido del edificio en un rango comprendido entre riesgo mínimo $=1$ y riesgo máximo $=3$, sin entrar en el análisis desglosado de las variables. Los resultados se muestran en la tabla 5 para cada experto y edificio analizado. Los datos reflejados en la tabla incluyen esta valoración de riesgo entre 1 y 3 , así como cuantía del riesgo total del edificio obtenido en la etapa anterior, comprendido entre 36 y 109.
En la tabla 5 se observa una correspondencia entre el nivel de riesgo percibido por los expertos y el incorporado en el diseño de las características de los edificios: la media de riesgo en las valoraciones de los expertos fue de 1,14 en el edificio 1 y 2,682,58-2,50 en los restantes. El valor de 1,14 se sitúa en el entorno de riesgo bajo (valor $=1$ ) con el que se diseña el edificio 1 , y los valores de 2,68-2,58-2,50 se sitúan igualmente en el entorno de riesgo alto (valor $=3$ ) con el que se diseñan los edificios 2, 3 y 4 .

Tabla 5. Equivalentes numéricos de los niveles de riesgo global percibido-valor de riesgo del edificio

\begin{tabular}{|c|c|c|c|c|}
\hline \multirow{2}{*}{ Expertos } & \multicolumn{4}{|c|}{ Edificios } \\
\hline & $\mathbf{1}$ & 2 & 3 & 4 \\
\hline 1 & $\begin{array}{c}1 \\
44,46\end{array}$ & - & - & - \\
\hline 2 & $\begin{array}{c}1 \\
38,46 \\
\end{array}$ & $\begin{array}{c}3 \\
78,39 \\
\end{array}$ & $\begin{array}{c}2 \\
80,38\end{array}$ & - \\
\hline 3 & $\begin{array}{c}1,5 \\
40,11\end{array}$ & $\begin{array}{c}2,5 \\
84,49\end{array}$ & $\begin{array}{c}2,5 \\
78,34\end{array}$ & $\begin{array}{c}2,5 \\
80,00 \\
\end{array}$ \\
\hline 4 & $\begin{array}{c}1 \\
41,33 \\
\end{array}$ & $\begin{array}{c}3 \\
59,63\end{array}$ & - & - \\
\hline 5 & $\begin{array}{c}1 \\
44,46 \\
\end{array}$ & $\begin{array}{c}2,75 \\
64,71 \\
\end{array}$ & $\begin{array}{c}3 \\
75,19 \\
\end{array}$ & - \\
\hline 6 & $\begin{array}{c}1 \\
39,03\end{array}$ & $\begin{array}{c}2 \\
60,86\end{array}$ & $\begin{array}{c}3 \\
69,64\end{array}$ & $\begin{array}{c}2 \\
77,55\end{array}$ \\
\hline 7 & $\begin{array}{c}2 \\
40,55\end{array}$ & $\begin{array}{c}3 \\
65,07 \\
\end{array}$ & $\begin{array}{c}3 \\
65,26 \\
\end{array}$ & $\begin{array}{c}3 \\
64,01 \\
\end{array}$ \\
\hline 8 & $\begin{array}{c}1 \\
48,42 \\
\end{array}$ & $\begin{array}{c}2 \\
75,15 \\
\end{array}$ & $\begin{array}{c}3 \\
62,06 \\
\end{array}$ & - \\
\hline 9 & $\begin{array}{c}1 \\
39,14 \\
\end{array}$ & $\begin{array}{c}2,5 \\
91,29 \\
\end{array}$ & $\begin{array}{c}3 \\
89,90\end{array}$ & $\begin{array}{c}2,5 \\
86,50 \\
\end{array}$ \\
\hline 10 & - & - & $\begin{array}{c}1 \\
57,74\end{array}$ & $\begin{array}{c}2,5 \\
71,52 \\
\end{array}$ \\
\hline 11 & $\begin{array}{c}1 \\
44,43 \\
\end{array}$ & $\begin{array}{c}3 \\
67,77\end{array}$ & - & - \\
\hline 12 & - & - & $\begin{array}{c}2,75 \\
73,81 \\
\end{array}$ & - \\
\hline 13 & $\begin{array}{c}1 \\
40,42 \\
\end{array}$ & $\begin{array}{c}3 \\
87,37 \\
\end{array}$ & $\begin{array}{c}2,5 \\
83,79 \\
\end{array}$ & - \\
\hline Media & $\begin{array}{c}1,14 \\
41,89 \\
\end{array}$ & $\begin{array}{c}\mathbf{2 , 6 8} \\
73,47\end{array}$ & $\begin{array}{c}2,58 \\
73,61\end{array}$ & $\begin{array}{r}\mathbf{2 , 5 0} \\
75,92\end{array}$ \\
\hline
\end{tabular}

En la siguiente etapa de la investigación se comprueba la correlación existente entre los valores reflejados en la tabla 5 y se establece una correspondencia entre los posibles valores de riesgo de un edificio, situados en el rango de 36 a 109 y los niveles de riesgo expresados en lenguaje natural, situados en el rango de riesgo mínimo $=1$ y riesgo máximo $=3$.

\subsubsection{Determinación de la correspondencia entre valores y niveles de riesgo}

Para establecer la correspondencia valores-niveles de riesgo, se verifica previamente la relación existente entre ambas magnitudes, calculando el coeficiente de correlación de Paerson.

Las dos variables asociadas para el cálculo del coeficiente de correlación son:

$\mathrm{X}=$ Valor de riesgo del edificio obtenido tras las valoraciones de los expertos. Varía según las ecuaciones [1] y [2] entre 36 y 109.

$\mathrm{Y}=$ Nivel de riesgo percibido de forma global por los expertos en los edificios valorados. Varía entre bajo y alto, o sus equivalentes numéricos 1 y 3 respectivamente. 


\section{GRÁFICO DE RELACIÓN ENTRE VARIABLES}

Ecuación: $y=0,0351 x-0,1019$ - EDIFICIOS

$1,2,3$ y 4

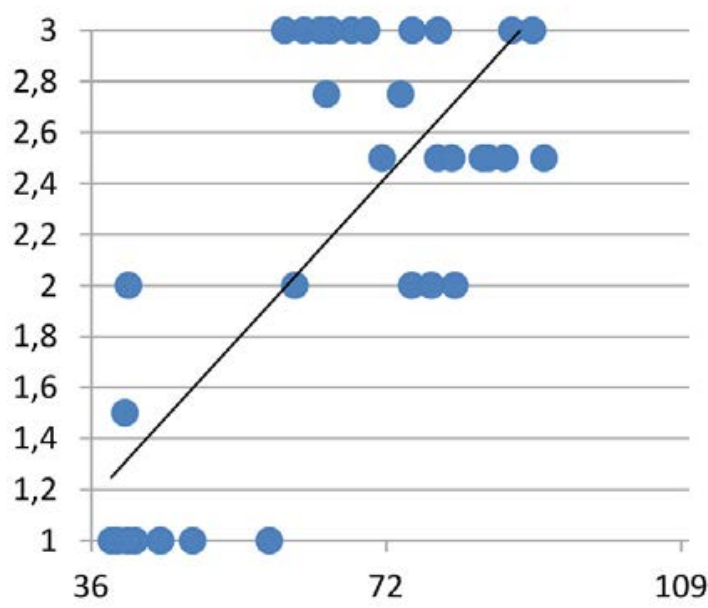

$\mathrm{X}=$ Riesgo del edificio calculado según modelo

Figura. 1. Nube de puntos y recta de regresión que relaciona los valores de riesgo calculado (X) y nivel de riesgo percibido de forma global en el edificio (Y).

En la tabla 5 se recogen los valores de X e Y. El coeficiente de correlación de Pearson obtenido mediante una hoja de cálculo Excel, fue de 0,75, que al encontrarse cercano a 1, puede considerarse que la correlación es fuerte. Por tanto, el conocimiento de la variable $\mathrm{X}$ dirá mucho acerca de la $\mathrm{Y}$.

Esta correlación permite hacer aproximaciones sobre el valor de una de las variables conociendo el valor de la otra, en concreto, poder asignar al edificio un nivel de riesgo situado entre bajo y alto, a partir de un valor de riesgo entre 36 y 109. Es decir, hasta este momento, la metodología desarrollada permite obtener una puntuación de valor de riesgo del edificio entre 36 y 109, pero se desconoce a qué nivel de riesgo corresponde entre bajo y alto.

Para establecer esta correspondencia valor-nivel de riesgo se obtiene la recta de regresión (figura 1) de la nube de puntos de los valores de X e Y. La ecuación [3] es la correspondiente a la recta de regresión:

$$
\mathrm{Y}=0,0351 \mathrm{X}-0,1019
$$

\subsubsection{Definición de la graduación correspondiente a las} opciones de nivel de riesgo total bajo, medio y alto

En esta etapa se establece la correspondencia entre los valores de riesgo de rango 36-109, y los niveles de riesgo comprendidos entre bajo $=1$ y alto $=3$. Al objeto de aportar información complementaria, la zona de riesgo medio $=2$ se subdivide en tres subni- veles: medio-bajo $=1,5 ;$ medio $=2$ y medio-alto $=2,5$. La amplitud de la zona de riesgo medio es de 1 punto (desde medio-bajo $=1,5$ a medio-alto=2,5), o o,33 puntos en cada subnivel. Por tanto, como la puntuación inicial de la zona de riesgo medio-bajo es 1,50; las puntuaciones finales de las zonas de riesgo medio-bajo, medio y medio-alto son $1,83-2,16-2,49$ respectivamente. A efectos prácticos se redondean a 1,85-2,15-2,50.

Para establecer la equivalencia entre los niveles de riesgo del edificio: bajo, medio-bajo, medio, medio-alto y alto (variable Y), y los valores de riesgo en la escala de 36 a 109 (variable X), se parte de la ecuación [3] de la recta de regresión obtenida de la nube de puntos generada por las variables X e Y. Partiendo de la variable $\mathrm{Y}$ de nivel de riesgo del edificio (ecuación [3]: $\mathrm{Y}=0,0351 \mathrm{X}-0,1019)$, cuyos equivalentes numéricos son conocidos: 1-1,5-1,85-2,15-2,5-3, se obtiene el valor de la variable X (ecuación [4]), cuyo rango de valores es 36-109:

$$
\mathrm{X}=\frac{(\mathrm{Y}+0,1019)}{0,0351}
$$

En la tabla 6 se indican los valores de la variable X correspondientes a los diferentes niveles de la variable Y. A efectos prácticos los valores de $\mathrm{X}$ se han redondeado según se indica en la tabla.

\begin{tabular}{|c|c|c|c|}
\hline$\underset{\mathbf{Y}}{\text { Valor }}$ & Valor X & {$[X=(Y+0,1019) / 0,0351]$} & \begin{tabular}{|c|} 
Valor $\mathrm{X}$ \\
adoptado
\end{tabular} \\
\hline 1,50 & & 45,64 & 45 \\
\hline 1,85 & & 55,61 & 55 \\
\hline 2,15 & & 64,16 & 65 \\
\hline 2,50 & & 74,13 & 75 \\
\hline
\end{tabular}

Tabla 6. Valores de X obtenidos a partir de la variable $\mathrm{Y}$

De esta manera, al calcular el riesgo de un edificio concreto y obtener una puntuación, o valor del riesgo entre 36 y 109 (variable $\mathrm{X}$ ), se obtiene la equivalencia de nivel de riesgo total del edificio (variable Y), según la matriz de correspondencia indicada en la tabla 7:

Tabla 7. Equivalencia valores de riesgo (variable $\mathrm{X}$ ) - niveles de riesgo (variable $\mathrm{Y}$ ).

\begin{tabular}{|c|c|}
\hline $\begin{array}{c}\text { Valor de riesgo (X) } \\
\text { (ecuaciones [1] y [2]) }\end{array}$ & Nivel de riesgo total (Y) \\
\hline 36 a 45 & Bajo \\
\hline 45 a 55 & Medio-Bajo \\
\hline 55 a 65 & Medio \\
\hline 65 a 75 & Medio-Alto \\
\hline 75 a 109 & Alto \\
\hline
\end{tabular}

La representación gráfica de la equivalencia de valores-niveles de riesgo se indica en la figura 2.

Nivel de riesgo del edificio

\begin{tabular}{|l|r|r|rrr|r|}
\hline BAJO & MEDIO-BAJO & MEDIO & MEDIO-ALTO & ALTO & \\
\hline 36 & 45 & 55 & 65 & 75 & 109
\end{tabular}

Valor de riesgo del edificio

Figura. 2. Representación gráfica equivalencia valores de riesgo-niveles de riesgo. 


\subsubsection{Implicaciones del nivel de riesgo del edificio}

El nivel de riesgo definido como bajo-medio-alto es un indicador sobre su magnitud y por tanto sobre la conveniencia de emprender acciones para modificarlo. De forma adicional a la información aportada por este indicador, en las entrevistas llevadas a cabo en la actividad $n^{\circ} 8$ (tabla 1), y tras el análisis profundo de las variables y las características de cada edificio, llevado a cabo por los participante en el proceso de aplicación del modelo, se solicita a los expertos información complementaria sobre el significado e implicaciones del nivel de riesgo. Estas informaciones ponen de manifiesto que un nivel bajo es en general propicio, aunque es necesario analizar si existen variables que impliquen riesgos a reducir. Un nivel medio es en general no deseable por la presencia de más riesgos que pueden requerir acciones más contundentes. El nivel alto es a priori inasumible en las condiciones iniciales, o asumible pero con unos incrementos de coste de posconstrucción con repercusiones previsiblemente elevadas. Pueden ser necesarias diferentes acciones profundas para reducir los riesgos.

\section{DISCUSIÓN DE RESULTADOS}

El proceso para la obtención del nivel de riesgo en un edificio consiste en: a) valoración del riesgo aportado por las variables, b) obtención del valor de riesgo del edificio mediante agregación ponderada de los anteriores valores, c) traducción de este valor numérico a un nivel de riesgo expresado en lenguaje natural. El modelo se ha ensayado con diferentes edificios, que fueron diseñados para estos experimentos con unos niveles de riesgo determinados. Las valoraciones de los expertos los han verificado y han puesto de manifiesto unos niveles de riesgo muy similares en los diferentes edificios ensayados de riesgo alto, los cuales han obtenido puntuaciones muy próximas a 75 .

El rango de los posibles valores de riesgo varía entre 36 y 109. Los diferentes niveles de riesgo comprendidos en este rango se han establecido siguiendo una metodología de trabajo que presenta una novedad frente a otras escalas y matrices indicativas de niveles del riesgo. Es habitual que los criterios que hacen equivaler resultados numéricos de valoración de riesgo con niveles de riesgo que expresan severidad, estén prefijados desde la fase inicial del proceso. En la presente investigación los niveles de severidad del riesgo se han obtenido a partir de un análisis e interpretación de resultados que relaciona los diferentes valores obtenidos tras aplicar la formulación del modelo con las opiniones de los expertos sobre el significado de dichos valores. Esto ha permitido identificar un valor de riesgo crítico equivalente a la puntuación 75 que se sitúa aproximadamente en el punto medio del rango entre 36 y 109. Este valor se traduce en un nivel de riesgo del edificio con unas repercusiones que se consideran inasumibles. Hasta este valor de riesgo crítico la escala de graduación presenta unos niveles de riesgo de amplitud uniforme.

Para determinar la necesidad de emprender acciones para reducir riesgos, hay que considerar que las técnicas de cuantificación del riesgo tienen por objetivo no solo cuantificar la probabilidad de fracaso, sino también entender el tipo de modo de fallo que se puede producir (31). Esto quiere decir que en el modelo propuesto hay dos ámbitos de criterios a considerar para determinar si es necesario emprender accio- nes para modificar los riesgos. El primer ámbito se refiere al nivel de riesgo reflejado en la escala de gradación. Según este criterio un nivel de riesgo inasumible, se alcanza con un valor de riesgo situado en el entorno de 75 y pone de manifiesto la necesidad de poner en marcha con contundencia diferentes tipos de medidas. El segundo ámbito de criterios se refiere al análisis pormenorizado de las variables, que es necesario llevar a cabo para identificar riesgos parciales que requieran medidas específicas para reducirlos.

Con referencia a una posible variabilidad de los resultados motivada por la participación de diferentes aplicadores del modelo, hay que indicar que como refiere a este respecto la norma ISO-31010:2009 (27), esta circunstancia no invalida una cuantificación realizada por especialistas conocedores de sus campos de actuación. Lejos de ser considerada como una limitación, la posible variabilidad en los juicios expertos permite obtener una perspectiva más completa de los riesgos, sirve de ayuda a la hora de asegurar que se identifican adecuadamente y aporta experiencia a la hora de planificar acciones para su tratamiento.

\section{CONCLUSIONES}

La presente investigación plantea una metodología para gestionar de forma previa a la fase de construcción, el riesgo de incrementos en la cuantía de los costes de la fase de uso del edificio. Esto supone un aporte respecto de la perspectiva tradicional en el análisis y control de costes en edificación, más centrada en la fase de construcción. El modelo permite detectar, anticipar y evitar incrementos de coste de posconstrucción, extendiendo así el concepto de sostenibilidad de la edificación desde la perspectiva ambiental a la social y económica. De esta manera se contribuye la creación de valor y la gestión eficiente de los edificios y se pone a disposición de los diferentes agentes implicados en la materialización, uso y explotación del edificio una herramienta que permite valorar diferentes opciones de proyecto. El manejo de esta herramienta no reviste complejidad, lo que contribuye a su aplicabilidad y a superar la brecha existente entre la teoría y la práctica de la evaluación del riesgo, especialmente en el sector de la edificación.

El valor de riesgo que se obtiene tras la aplicación de la metodología, adopta puntuaciones entre un mínimo 36 y un máximo de 109 que se traducen en un nivel de riesgo expresado en lenguaje natural que es un indicador del estado general de riesgo del edificio. Los experimentos de aplicación del modelo en los edificios estudiados han puesto de manifiesto un nivel de riesgo crítico del edificio correspondiente a un valor de riesgo de 75. Este nivel de riesgo crítico supone unos incrementos de coste de posconstruccion que se consideran inasumibles, lo que implica tener que llevar a cabo cambios en las características del proceso de materialización del edificio a las que hacen referencia las variables de riesgo contempladas. En esta línea de determinar la necesidad de poner en práctica las acciones específicas para reducir los riesgos, el proceso de aplicación de la metodología y el desglose en variables permiten desarrollar una comprensión del origen y las causas de los incrementos de coste de posconstruccción.

Entre las variables de riesgo que forman parte del modelo es de destacar por los índices marcados por los expertos, la importancia de los acabados, materiales y sistemas construc- 
tivos a emplear en las fachadas del edificio, la planificación de un mantenimiento posterior del edificio enfocado a reducir acciones correctivas, así como la idoneidad técnica de los agentes que intervienen en el diseño, dirección y ejecución de las obras.

El procedimiento metodológico seguido para determinar las variables y su ponderación emplea un método Delphi, en el que se han sustituido los formularios de consulta por entrevistas semiestructuradas, las cuales han aumentado la calidad de los datos y han permitido integrar en una escala de medida común, múltiples opiniones expertas acerca del significado de los valores de riesgo calculados.
El modelo es adaptable a otros tipos de edificaciones, usos o ámbitos geográficos ajustando la relación valor de riesgonivel de riesgo, así como los principales parámetros que forman parte de la herramienta propuesta: relación de variables y sus ponderaciones, lo que puede ser objeto de trabajos posteriores.

\section{AGRADECIMIENTOS}

Los autores quieren dejar constancia de su agradecimiento a los expertos participantes en la investigación, tanto externos como pertenecientes a la Escuela Técnica Superior de Ingeniería de Edificación de Sevilla.

\section{REFERENCIAS}

(1) García-Erviti, F., Armengot-Paradinas, J., Ramírez-Pacheco, G. (2015). El análisis del coste del ciclo de vida como herramienta para la evaluación económica de la edificación sostenible. Estado de la cuestión. Informes de la Construcción, 67(537): e056, doi: http://dx.doi.org/10.3989/ic.12.119.

(2) ISO (2014). ISO 55000:2015 Asset management -- Overview, principles and terminology. International Organization for Standardization.

(3) ISO (2008). ISO 15686-5:2008(E) Buildings and constructed assets - Service-life planning - Part 5: Life-cycle costing. International Organization for Standardization.

(4) Baloi, D., Price, A.D.F. (2003). Modelling global risk factors affecting construction cost performance. International Journal of Project Management, 21: 261-269, doi: https://doi.org/10.1016/So263-7863(02)00017-0.

(5) PMI. (2017). A Guide to the Project Management Body of Knowledge: (PMBOK® Guide). Sixth ed., Newtown Square, PA: PMI, Project Management Institute.

(6) Fernández-Valderrama, P. (2015). Modelo para la gestión del riesgo asociado a los costes globales en fase de postconstrucción en edificios plurifamiliares destinados a arrendamiento (tesis doctoral). Universidad de Sevilla. Sevilla, España.

(7) Zhou, L., Vasconcelos, A., Nunes, M. (2008). Supporting decision making in risk management through an evidencebased information systems project risk checklist. Information management and computer security, 16(2), 166-186, doi: https://doi.org/10.1108/09685220810879636.

(8) Uher, T.E., Toakley, A. (1999). Risk management in the conceptual phase of a project. International Journal of Project Management, 17: 161-169, doi: https://doi.org/10.1016/So263-7863(98)ooo24-6.

(9) Han, S.H., Kim, D.Y., Kim, H. and Jang, W.S. (2008). A web-based integrated system for international project risk management. Automation in Construction, 17: 342-356, doi: https://doi.org/10.1016/j.autcon.2007.05.012.

(10) Shang, H., Anumba, C.J., Bouchlaghem, D.M., Miles, J.C., Cen, M., Taylor, M. (2005). An intelligent risk assessment system for distributed construction teams. Engineering Construction and Architectural Management, 12: 391-409, doi: https://doi.org/10.1108/09699980510608839.

(11) Ministerio de Fomento (2013). Real Decreto 233/2013, de 5 de abril, por el que se regula el Plan Estatal de fomento del alquiler de viviendas, la rehabilitación edificatoria, y la regeneración y renovación urbanas, 2013-2016. Boletín Oficial del Estado, n ${ }^{0}$ 10. España.

(12) Ministerio de Fomento (2018). Real Decreto 106/2018, de 9 de marzo, por el que se regula el Plan Estatal de Vivienda 2018-2021. Boletín Oficial del Estado, nº 61. España.

(13) Taroun, A. (2014). Towards a better modelling and assessment of construction risk: Insights from a literature review. International Journal of Project Management, 32: 101-115, doi: https://doi.org/10.1016/j.ijproman.2013.03.004.

(14) Akintoye, A.S., MacLeod, M.J. (1997). Risk analysis and management in construction. International Journal of Project Management, 15(1): 31-38, doi: https://doi.org/10.1016/So263-7863(96)ooo35-X.

(15) De Camprieu, R., Desbiens, J., Feixue, Y. (2007). 'Cultural' differences in project risk perception: An empirical comparison of China and Canada. International Journal of Project Management, 25(7): 683-693, doi: https://doi.org/10.1016/j. ijproman.2007.07.005.

(16) Hwang, B.G., Zhao, X., Toh, L.P. (2014). Risk management in small construction projects in Singapore: Status, barriers and impact. International Journal of Project Management, 32(1): 116-124, doi: https://doi.org/10.1016/j.ijproman.2013.01.007.

(17) Marcelino-Sádaba, S., Pérez-Ezcurdia, A., Lazcano, A.M.E., Villanueva, P. (2014). Project risk management methodology for small firms. International Journal of Project Management, 32(2): 327-340, doi: https://doi.org/10.1016/j. ijproman.2013.05.009.

(18) Osipova, E., Eriksson, P.E. (2013). Balancing control and flexibility in joint risk management: Lessons learned from two construction projects. International Journal of Project Management, 3(3): 391-399, doi: https://doi.org/10.1016/j. ijproman.2012.09.007.

(19) Bjerga, T., Aven, T. (2015). Adaptive risk management using new risk perspectives-an example from the oil and gas industry. Reliability Engineering and System Safety, 134: 75-82, doi: https://doi.org/10.1016/j.ress.2014.10.013. 
(20) Aven, T., Krohn, B.S. (2014). A new perspective on how to understand, assess and manage risk and the unforeseen. Reliability Engineering and System Safety, 121: 1-10, doi: https://doi.org/10.1016/j.ress.2013.07.005.

(21) Kuo, Y.C., Lu, S.T. (2013). Using fuzzy multiple criteria decision making approach to enhance risk assessment for metropolitan construction projects. International Journal of Project Management, 31(4): 602-614, doi: https://doi. org/10.1016/j.ijproman.2012.10.003.

(22) Rostami, A., Oduoza, C. F. (2017). Key risks in construction projects in Italy: contractors' perspective. Engineering, Construction and Architectural Management, 24(3): 451-462, doi: https://doi.org/10.1108/ECAM-09-2015-0142.

(23) Likert, R. (1932). A technique for the measurement of attitudes. Archives of psychology, 22(140): 1-55.

(24) Lu, S., Yan, H. (2013). A comparative study of the measurements of perceived risk among contractors in China. International Journal of Project Management, 3: 307-312, doi: https://doi.org/10.1016/j.ijproman.2012.06.001.

(25) Dawson, I.G., Johnson, J.E., Luke, M.A. (2017). One too many? Understanding the influence of risk factor quantity on perceptions of risk. Risk analysis, 37(6): 1157-1169, doi: https://doi.org/10.1111/risa.12690.

(26) Kangari, R., Riggs, L. S. (1989). Construction risk assessment by linguistics. Engineering Management, IEEE Transactions on, 36 (2): 126-131, doi: https://doi.org/10.1109/17.18829.

(27) ISO (2010). ISO 31010: 2009 Risk Management-Risk Assessment Techniques. CENELEC.

(28) Cervone, H.F. (2006). Project risk management. OCLC Systems and Services, 22: 256-262, doi: https://doi. org/10.1108/10650750610706970.

(29) Kendrick, T. (2003). Identifying and Managing Project Risk, New York: American Management Association.

(30) Nassim, N.T. (2007). The black swan: the impact of the highly improbable. New York: Random House.

(31) Apostolakis, G.E. (2004). How useful is quantitative risk assessment? Risk Analysis, 24: 515-520, doi: https://doi. org/10.1111/j.0272-4332.2004.00455.x. 